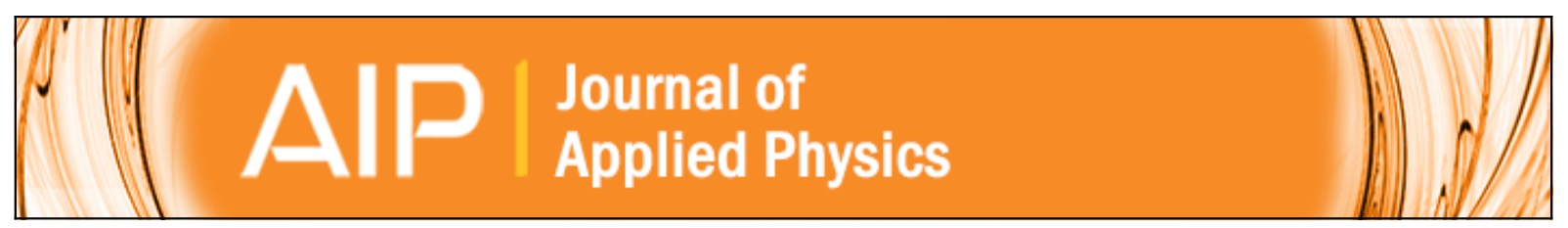

Co-doping with antimony to control phosphorous diffusion in germanium

H. A. Tahini, A. Chroneos, R. W. Grimes, and U. Schwingenschlögl

Citation: Journal of Applied Physics 113, 073704 (2013); doi: 10.1063/1.4792480

View online: http://dx.doi.org/10.1063/1.4792480

View Table of Contents: http://scitation.aip.org/content/aip/journal/jap/113/7?ver=pdfcov

Published by the AIP Publishing

AlPP Re-register for Table of Content Alerts

Create a profile.

Sign up today! 


\title{
Co-doping with antimony to control phosphorous diffusion in germanium
}

\author{
H. A. Tahini, ${ }^{1}$ A. Chroneos, ${ }^{2, a)}$ R. W. Grimes, ${ }^{1}$ and U. Schwingenschlögl ${ }^{3}$ \\ ${ }^{1}$ Department of Materials, Imperial College London, London SW7 2AZ, United Kingdom \\ ${ }^{2}$ Materials Engineering, The Open University, Milton Keynes MK7 6AA, United Kingdom \\ ${ }^{3}$ PSE Division, KAUST, Thuwal 23955-6900, Saudi Arabia
}

(Received 9 January 2013; accepted 1 February 2013; published online 15 February 2013)

\begin{abstract}
In germanium, phosphorous and antimony diffuse quickly and as such their transport must be controlled in order to design efficient $n$-typed doped regions. Here, density functional theory based calculations are used to predict the influence of double donor co-doping on the migration activation energies of vacancy-mediated diffusion processes. The migration energy barriers for phosphorous and antimony were found to be increased significantly when larger clusters involving two donor atoms and a vacancy were formed. These clusters are energetically stable and can lead to the formation of even larger clusters involving a number of donor atoms around a vacancy, thereby affecting the properties of devices. (C) 2013 American Institute of Physics. [http://dx.doi.org/10.1063/1.4792480]
\end{abstract}

\section{INTRODUCTION}

Germanium $(\mathrm{Ge})$ is emerging as an important material for nanoelectronic devices, not only due to its superior properties (lower dopant activation temperatures, smaller band gap, and high carrier mobilities) but also due to the realization of high dielectric constant (high- $k$ ) dielectrics on top of group IV semiconductors. ${ }^{1}$ Although $\mathrm{Si}$ and Ge are isostructural, the vacancy is the dominant intrinsic point defect, that is, only in Ge. ${ }^{2}$ Vacancies can have a deleterious impact on the fabrication of high performance Ge-metal-oxide-semiconductor field-effect transistor (MOSFET) or other Ge based devices. ${ }^{3}$ In particular, the channel region of the device vacancies provide scattering centers degrading the carrier mobility; the source and drain junctions act as recombination sites increasing the leakage current. Finally, the $n$-type regions vacancies can enhance donor atom diffusion or cause the deactivation of donor atoms via cluster formation. ${ }^{3,4}$

It is important to constrain or reduce the concentration of vacancies in Ge as they are the vehicle mediating donor atom diffusion and also the important constituent of the clusters related to deactivation (for example, $\mathrm{As}_{\mathrm{n}} V_{\mathrm{m}}$ or $\mathrm{Sb}_{\mathrm{n}} V_{\mathrm{m}}$ clusters). ${ }^{5}$ To control vacancies, a number of point defect engineering strategies have been proposed. ${ }^{3,4,6-8}$ A study of density functional theory (DFT) on P and As co-doping proposed that double-donor doping can be an effective way to engineer the active donor concentrations. ${ }^{6}$ That study inspired the experimental investigation of Tsouroutas et al. ${ }^{7}$ on $\mathrm{P}$ and As co-doping. Tsouroutas et al., ${ }^{7}$ however, concluded that although there is a retardation of As diffusion, the activation level of co-doped Ge is lower compared to the singly doped Ge. Recently, Kim et al. ${ }^{8}$ used $\mathrm{P}$ and $\mathrm{Sb}$ codoping to improve $\mathrm{Ge} \mathrm{n}^{+} / \mathrm{p}$ junction diode characteristics. They concluded that $\mathrm{P}$ and $\mathrm{Sb}$ co-doping has a beneficial impact upon the $n$-type dopant activation leading to an increased forward biased current density in shallow junction diodes. Kim et al. ${ }^{8}$ hypothesise that the mechanism for the enhanced activation in $\mathrm{P}$ and $\mathrm{Sb}$ co-doped $\mathrm{Ge}$ might be

\footnotetext{
a) Author to whom correspondence should be addressed. Electronic mail: Alex.Chroneos@open.ac.uk.
}

linked to local strain compensation ( $\mathrm{P}$ is smaller than $\mathrm{Ge}$, whereas $\mathrm{Sb}$ is larger). The aim of the present study is to quantify the binding of $\mathrm{P}$ and $\mathrm{Sb}$ to vacancies and the impact of co-doping on the migration energy of these donor atoms in $\mathrm{Ge}$.

\section{METHODOLOGY}

DFT implemented numerically in the Vienna $a b$ initio Simulation Package (VASP) that was used to calculate the defect energetics (binding energies, migration energies). ${ }^{9}$ The generalised gradient approximation (GGA) with the PerdewBurke-Ernzerhof (PBE) description of the electron and exchange correlation was used. ${ }^{10} \mathrm{Ge}, \mathrm{P}$, and $\mathrm{Sb}$ were described using $4 s^{2} 4 p^{2}, 3 s^{2} 3 p^{3}$, and $5 s^{2} 5 p^{3}$ pseudopotentials generated using the projector augmented wave (PAW) approach. ${ }^{11} \mathrm{~A}$ $2 \times 2 \times 2$ Monkhorst-Pack mesh was used to sample the Brillouin zone. ${ }^{12}$ Energies and forces were iterated until an accuracy of $1 \times 10^{-5} \mathrm{eV}$ and $1 \times 10^{-3} \mathrm{eV} / \AA$ were achieved, respectively. Migration energy barriers were calculated using the climbing image nudged elastic band (CI-NEB) technique. ${ }^{13}$ All simulations were carried out at constant volume using the calculated perfect lattice parameter of Ge $(5.5985 \AA)$ in a 216 atom supercell. Electron spin-polarisation was accounted in the simulations. DFT underestimates the band gap of Ge. In order to correct this, we used a GGA $+U$ approaches implemented previously, which was positively compared to the performance of the HSE06 hybrid functional. ${ }^{14-16}$ Charged defect clusters were created by adding or removing electrons from the simulation supercell. The 216 atoms supercell was chosen to minimise the effect of the charged defect-defect interactions. In addition, NEB results are taken as the differences between successive images leading to the cancelation of spurious interactions.

\section{RESULTS AND DISCUSSION}

As established by Brotzmann and Bracht, ${ }^{2}$ the strong doping dependence in phosphorous-doped $\mathrm{Ge}$ can be explained by the formation of negatively charged phosphorous-vacancy, $(\mathrm{PV})^{-}$, pairs via the reaction,

$$
P_{s}^{+}+V^{2-} \leftrightarrow(P V)^{-1} .
$$



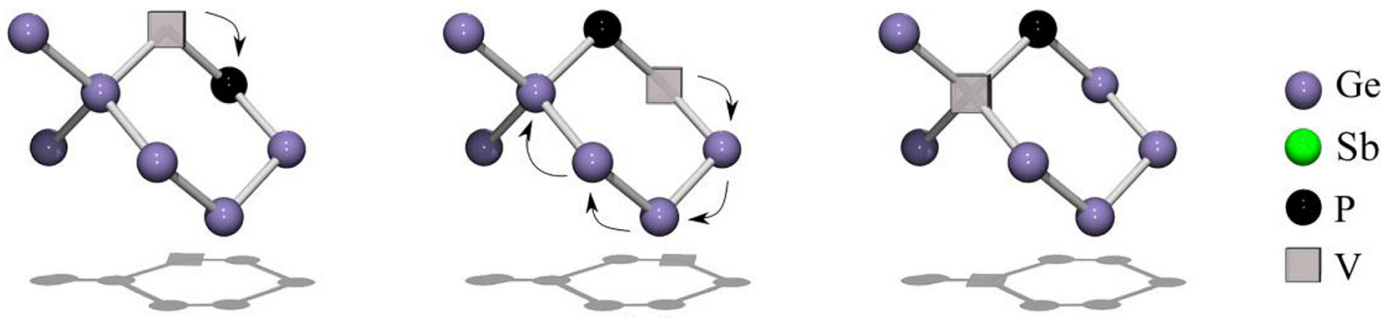

(a)
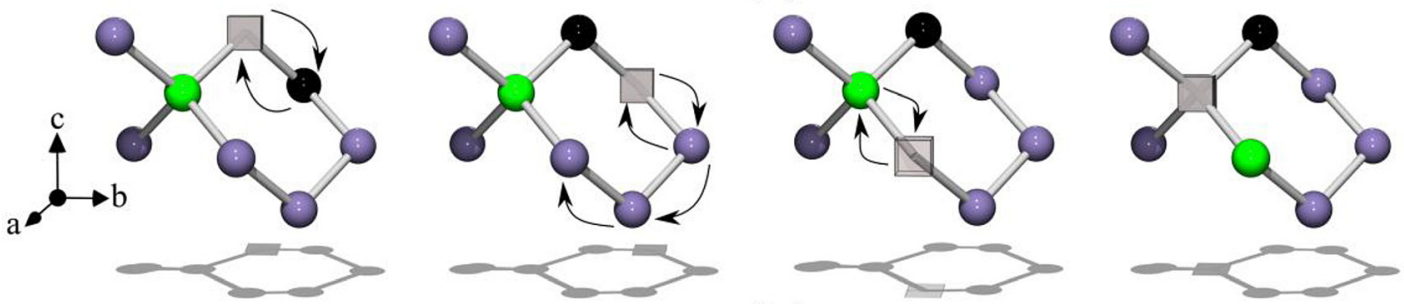

(b)

FIG. 1. A schematic representation of (a) the diffusion of $\mathrm{P}$ via the ring-mechanism of diffusion of a $\mathrm{P}$ in the Ge lattice. In this mechanism the $V$ exchanges position with the $\mathrm{P}$ atom and then it progresses through the ring approaching the $\mathrm{P}$ atom through another direction. (b) Represents the proposed ring mechanism of diffusion where the $\mathrm{P} V$ pair encounters a $\mathrm{Sb}$ atom.

Where $P_{s}^{+}$denotes the single positively charged P substitutional atom and $V^{2-}$ the double negatively charged vacancy. The key to controlling $\mathrm{P}$ diffusion in $\mathrm{Ge}$ is the control of the vacancies, as these progress the fast diffusion process.

We first investigate the migration energy of the single negatively charged pair $(\mathrm{PV})^{-1}$. It has been previously established that the $V$-mediated diffusion of dopants in Ge proceeds $v i a$ the ring mechanism of diffusion. ${ }^{2}$ Fig. 1(a) shows the ring diffusion mechanism for the (PV $)^{-1}$ cluster and Fig. 2(a) the energies associated with each step. The migration energy barrier (i.e., the step along the ring with the highest energy barrier in Fig. 2(a)) for the single negatively charged pair $(\mathrm{PV})^{-1}$ is $0.91 \mathrm{eV}$. For completeness the migration energy barrier of the $(\mathrm{Sb} V)^{-1}$ pair was also calculated $(1.17 \mathrm{eV})$.

To consider the trapping of the $(\mathrm{P} V)^{-1}$ pair to further donor atoms ( $\mathrm{P}$ or $\mathrm{Sb})$, we consider the following reactions:

$$
(P V)^{-1}+P_{s}^{+} \leftrightarrow P_{2} V \text { and }(P V)^{-1}+S b_{s}^{+} \leftrightarrow P S b V .
$$

The formation of these neutral clusters is promoted by the attraction of the oppositely charged components. The $\mathrm{PSb} V$ cluster is more bound compared to the $\mathrm{P}_{2} V$ cluster $(-2.03 \mathrm{eV}$ and $-1.83 \mathrm{eV}$, respectively) because the oversized $\mathrm{Sb}$ atom benefits more from the relaxation near the vacant space of the $(\mathrm{PV})^{-1}$ pair. This is also indicated by the binding energies of the $(\mathrm{SbV})^{-1}$ and $(\mathrm{PV})^{-1}$ pairs, which are $-1.19 \mathrm{eV}$ and $-0.93 \mathrm{eV}$, respectively. Interestingly, for the $(\mathrm{SbV})^{-1}$ pair, the oversized $\mathrm{Sb}$ atom occupies the space between two semi-vacant lattice sites, leading to a configuration known as the split-vacancy configuration. ${ }^{17}$ The present study reproduces this configuration in agreement with the previous DFT study of Höhler et al. ${ }^{17}$ Importantly, the difference in binding energies, in favour of the $\mathrm{Sb}$ atom, reveals that it will have a greater "radius of influence" to attract migrating $(\mathrm{PV})^{-1}$ pairs as compared to $\mathrm{P}$ atoms.

The next issue that needs to be considered is the mobility of the $\mathrm{P}_{2} V$ and $\mathrm{PSb} V$ clusters. Figs. 2(b) and 2(c) show
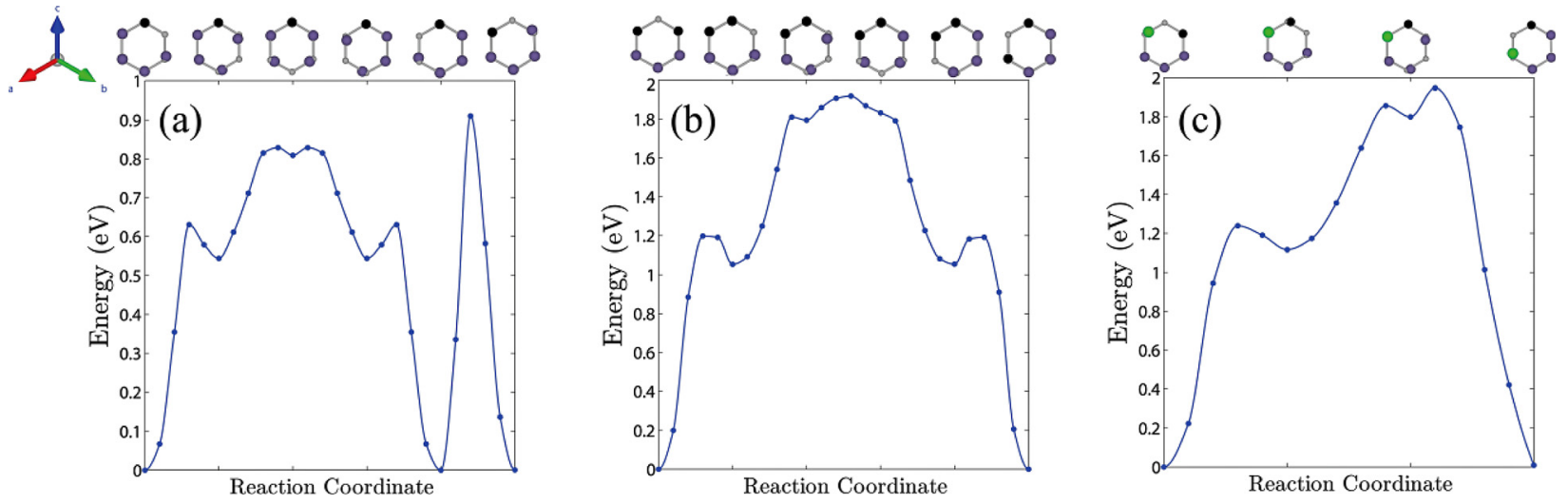

FIG. 2. Diffusion path of (a) the PV pairs (mechanism of Fig. 1(a)) and the PV pairs in the presence of (b) a second $\mathrm{P}$ atom and (c) an Sb atom (both mechanism of Fig. 1(b)). On the top of the figures is the ring mechanism of diffusion projected onto the (111) surface of Ge. The squares represent the $V$, smaller circles the $\mathrm{P}$ atom, and larger circles the $\mathrm{Sb}$. 
the energies of the diffusion paths for the $\mathrm{P}_{2} V$ and PSb $V$ clusters along the ring mechanism (Fig. 1(b)). The $\mathrm{P}_{2} V$ and PSbV clusters have migration energy barriers $1.92 \mathrm{eV}$ and $1.94 \mathrm{eV}$, respectively, indicating that there is practically no difference. Both these migration energy barriers are higher by more than $1 \mathrm{eV}$ compared to the $(\mathrm{PV})^{-1}$ pair and therefore the association of the pair with a further donor atom via Eq. (2) will lead to it being less mobile. For the $\mathrm{P}_{2} V$ pair, this was previously established by the study of Brotzmann et al., ${ }^{4}$ however, for mixed clusters there has been no such information. As mentioned earlier, Kim et al. ${ }^{8}$ hypothesised that the mechanism for enhanced activation in $\mathrm{P}$ and $\mathrm{Sb}$ co-doped $\mathrm{Ge}$ is linked to local strain compensation. The present study quantifies that mixed clusters such as PSb $V$ are more bound than $\mathrm{P}_{2} V$ (by $-0.2 \mathrm{eV}$ ); however, we also find that such larger clusters $\left(\mathrm{P}_{2} V\right.$ and/or PSbV) will be less mobile compared to the $(\mathrm{PV})^{-1}$ pair.

Next we consider how these binding and migration energies compare with $\mathrm{P}$ co-doped with an isovalent dopant. In a recent DFT study, it was shown that the binding energy of the $(\mathrm{PHfV})^{-1}$ cluster is $-3.55 \mathrm{eV}$ and therefore more energetically favourable compared to the PSbV cluster by about $1.5 \mathrm{eV}$. This in turn implies that Hf will be more effective than $\mathrm{Sb}$ in trapping the $(\mathrm{PV})^{-1}$ pair; however, the singly positively charged $\mathrm{Sb}$ atom can have an advantage in attracting the negatively charged $(\mathrm{PV})^{-1}$ pair via Coulombic interaction (and as such it is expected to have a larger radius of influence).

At this point one needs to consider whether the $(\mathrm{PHfV})^{-1}$ or the $\mathrm{PSb} V$ clusters would be more efficient precursors for the formation of the larger P-vacancy clusters that could lead to the deactivation of a significant part of the $\mathrm{P}$ dose. Considering that the $(\mathrm{PHfV})^{-1}$ clusters are singly negatively charged it is unlikely that they will attract the migrating $(\mathrm{PV})^{-1}$ pairs or the doubly negatively charged vacancies. They are also practically immobile as they have high migration energies. Conversely, the $\mathrm{P}_{2} V$ and PSbV clusters are neutral and thus larger clusters may still be formed as predicted via DFT/mass action analysis approaches (refer to Chroneos et al..$^{5}$ ). Notably, as the PSbV cluster is more bound compared to the $\mathrm{P}_{2} V$ cluster it may be deduced that in the case of codoping with $\mathrm{Sb}$ stronger deactivation should be expected. The formation of larger clusters is also supported by experimental work in $n$ type doped $\mathrm{Si}^{18}$ and $\mathrm{Ge}$ (Bruno et al. ${ }^{5}$ ).

The recent study of Schneider and Bracht ${ }^{19}$ highlighted that a supersaturation of Ge interstitials can suppress the formation of dopant-vacancy clusters. This approach is thus advantageous to maximise the activation level of donor atoms in implanted Ge. ${ }^{19}$ The difference of single P-doped Ge with $\mathrm{P}$ and $\mathrm{Sb}$ co-doped Ge maybe in part reconciled by the differences in the defect structure established under implantation and the subsequent rapid thermal annealing. The impact of Ge-interstitial in these processes needs to be detailed in future theoretical work.

\section{CONCLUSIONS}

A DFT/GGA $+U$ approach has been used to quantify the influence of co-doping and the formation of $\mathrm{PSb} V$ clusters on the activation energy for migration of $\mathrm{P}$ or $\mathrm{Sb}$ in $\mathrm{Ge}$. It is predicted that the formation of strongly bound $\mathrm{PSb} V$ clusters, results in the traping of the $(\mathrm{PV})^{-1}$ pair and retards its transport by increasing the migration activation energy. The present study is consistent with previous experimental studies but the exact mechanism of donor-vacancy cluster formation and deactivation in Ge needs to be further clarified.

\section{ACKNOWLEDGMENTS}

This publication was based on research supported by King Abdullah University for Science and Technology (KAUST). Computing resources were provided by the Shaheen supercomputer at KAUST (http://www.hpc.kaust.edu. $\mathrm{sa} /$ ) and the High Performance Computing (HPC) facility of Imperial College London (http://www3.imperial.ac.uk/ict/ services/highperformancecomputing).

${ }^{1}$ C. Claeys and E. Simoen, Germanium-Based Technologies: From Materials to Devices (Elsevier, 2007); A. Chroneos, J. Appl. Phys. 105, 056101 (2009); E. Napolitani, G. Bisognin, E. Bruno, M. Mastronatteo, G. G. Scapellato, S. Boninelli, D. De Salvador, S. Mirabella, C. Spinella, A. Carnera, and F. Priolo, Appl. Phys. Lett. 96, 201906 (2010); A. Chroneos, J. Appl. Phys. 107, 076102 (2010); H. Wang, A. Chroneos, A. Dimoulas, and U. Schwingenschlögl, Phys. Chem. Chem. Phys. 14, 14630 (2012).

${ }^{2}$ S. Brotzmann and H. Bracht, J. Appl. Phys. 103, 033508 (2008); A. Chroneos, H. Bracht, R. W. Grimes, and B. P. Uberuaga, Appl. Phys. Lett. 92, 172103 (2008).

${ }^{3}$ G. Impellizzeri, S. Boninelli, F. Priolo, E. Napolitani, C. Spinella, A. Chroneos, and H. Bracht, J. Appl. Phys. 109, 113527 (2011); W. S. Jung, J. H. Park, A. Nainani, D. Nam, and K. C. Saraswat, Appl. Phys. Lett. 101, 072104 (2012); A. Chroneos, Phys. Stat. Solidi B 244, 3206 (2007).

${ }^{4}$ S. Brotzmann, H. Bracht, J. L. Hansen, A. N. Larsen, E. Simoen, E. E. Haller, J. S. Christensen, and P. Werner, Phys. Rev. B 77, 235207 (2008); A. Chroneos, R. W. Grimes, B. P. Uberuaga, and H. Bracht, ibid. 77, 235208 (2008); A. Chroneos, R. W. Grimes, and H. Bracht, J. Appl. Phys. 106, 063707 (2009); E. Simoen and J. Vanhellemont, ibid. 106, 103516 (2009); H. Tahini, A. Chroneos, R. W. Grimes, U. Schwingenschlögl, and H. Bracht, Phys. Chem. Chem. Phys. 15, 367 (2013).

${ }^{5}$ A. Chroneos, R. W. Grimes, B. P. Uberuaga, S. Brotzmann, and H. Bracht, Appl. Phys. Lett. 91, 192106 (2007); E. Bruno, G. G. Scapellato, A. La. Magna, M. Cuscuna, E. Napolitani, S. Boninelli, F. Priolo, G. Fortunato, and V. Privitera, ibid. 101, 172110 (2012).

${ }^{6}$ A. Chroneos, R. W. Grimes, H. Bracht, and B. P. Uberuaga, J. Appl. Phys. 104, 113724 (2008).

${ }^{7}$ P. Tsouroutas, D. Tsoukalas, and H. Bracht, J. Appl. Phys. 108, 024903 (2010).

${ }^{8}$ J. Kim, S. W. Bedell, and D. K. Sadana, Appl. Phys. Lett. 98, 082112 (2011).

${ }^{9}$ G. Kresse and D. Joubert, Phys. Rev. B 59, 1758 (1999).

${ }^{10}$ J. P. Perdew, M. Ernzerhof, and K. Burke, J. Chem. Phys. 105, 9982 (1996).

${ }^{11}$ P. E. Blöchel, Phys. Rev. B 50, 17953 (1994).

${ }^{12}$ H. J. Monkhorst and J. D. Pack, Phys. Rev. B 13, 5188 (1976).

${ }^{13}$ G. Henkelman, B. P. Uberuaga, and H. Jónsson, J. Chem. Phys. 113, 9901 (2000).

${ }^{14}$ H. Tahini, A. Chroneos, R. W. Grimes, U. Schwingenschlögl, and H. Bracht, Appl. Phys. Lett. 99, 072112 (2011); 99, 162103 (2011); J. Phys.: Condens. Matter 24, 195802 (2012).

${ }^{15}$ J. Heyd, E. G. Scuseria, and M. Ernzerhof, J. Chem. Phys. 118, 8207 (2003).

${ }^{16}$ M. Marsman, J. Paier, A. Stroppa, and G. Kresse, J. Phys.: Condens. Matter 20, 064201 (2008).

${ }^{17}$ H. Höhler, N. Atodiresei, K. Schroeder, R. Zeller, and P. Dederichs, Phys. Rev. B 71, 35212 (2005).

${ }^{18}$ S. Solmi and D. Nobili, J. Appl. Phys. 83, 2484 (1998); J. Xie and S. P. Chen, J. Phys.: Condens. Matter 11, 7219 (1999); V. Ranki and K. Saarinen, Physica B 340-342, 765 (2003).

${ }^{19}$ S. Schneider and H. Bracht, Appl. Phys. Lett. 98, 014101 (2011). 